\title{
Effect of Constant Rate Infusion of Xylazine \& Xylazine Morphine Combination on Sedation, Analgesia and Ataxia in Horses
}

\author{
Amal Hammad*, Shaaban Gadallah, Ahmed Sharshar and Tarik Misk \\ Department of Surgery, Anesthesiology and Radiology, Faculty of Veterinary Medicine, University of Sadat city, Egypt
}

*Corresponding author: Amal Hammad, Department of Surgery, Anesthesiology and Radiology, Faculty of Veterinary Medicine, University of Sadat city, Egypt

To Cite This Article: Amal Hammad, Effect of Constant Rate Infusion of Xylazine \& Xylazine Morphine Combination on Sedation, Analgesia and Ataxia in Horses. 2020 - 8(3). AJBSR.MS.ID.001263. DOI: 10.34297/AJBSR.2020.08.001263

Received: March 09, 2020; Published: March 19, 2020

\begin{abstract}
In standing horses, notable sedation and analgesia may be attained by using $\alpha_{2}$-agonists/opioids combinations relative to $\alpha_{2}$-agonists alone. Therefore, this study aimed to evaluate the effect of a constant rate infusion (CRI) of xylazine (XYL) alone or in combination with morphine (XYL/ MOR) on sedation, analgesia and ataxia in horses. Five adult healthy horses were assigned to receive two sedative protocols at one week interval as follows, XYL ( $0.55 \mathrm{mg} / \mathrm{kg}$ of xylazine IV directly followed by xylazine CRI of $1.08 \mathrm{mg} / \mathrm{kg} / \mathrm{hour}$ followed later by saline bolus and a CRI of saline) and XYL/MOR $(0.55 \mathrm{mg} / \mathrm{kg}$ of xylazine IV directly followed by xylazine CRI of $0.66 \mathrm{mg} / \mathrm{kg} /$ hour followed later by morphine $0.15 \mathrm{mg} / \mathrm{kg}$, IV and morphine CRI of $0.1 \mathrm{mg} / \mathrm{kg} /$ hour). The effect of both protocols on sedation, analgesia and ataxia was assessed following xylazine administration by 10,15 minutes and later at 15 minutes interval for 120 minutes during infusion of the tested combinations and every 15 minutes for 60 minutes after cessation of infusion. Relative to baseline, significant changes in sedative and analgesic scores were detected for 120 minutes with significant ataxia for only 15 minutes following both treatments. Addition of morphine to xylazine produced significantly higher sedation scores compared to xylazine alone for 120 minutes with slightly higher analgesic scores at some time points without significantly different ataxia scores in between. In conclusion, xylazine/morphine protocol had a superior sedative effect compared to xylazine alone consequently it might be better alternative to xylazine protocol during standing interventions in horses.
\end{abstract}

Keywords: Xylazine; Morphine; Sedation; Analgesia; Ataxia; Horses

Abbreviations: CRI: Constant Rate Infusion; MOR: Morphine; XYL: Xylazine; NRS: Numerical Rating Scale; MFSS: Multifactorial Sedation Scale; ANOVA: One-Way Analysis of Variance

\section{Introduction}

Virtually, standing sedation is a prominent approach in equine practice whereas it eliminates the risks and expenses associated with general anesthesia and provides a suitable option for veterinarians to conduct various diagnostic and surgical procedures in horses with a deteriorated physical status [1-4]. $\alpha_{2}$-adrenoceptor agonists are routinely used during different standing procedures but sedation produced by these drugs alone is less reliable as the sedated horses may be aroused and respond unexpectedly to imposed stimuli especially to touch which represents a danger both to the horse and personnel involved [5-8]. For this, many authors recommended the use of $\alpha_{2}$-agonists in combination with opioids to improve their sedative and analgesic effects and to prolong their duration of action [9-11].
Furthermore, due to the dose-dependent side effects of $\alpha_{2}$ agonists, administration of opioids with them was proposed to reduce their required doses [12]. Standing sedation for prolonged duration can be conducted by administration of $\alpha_{2}$-agonists either as repeated boli or as constant rate infusion (CRI). Application of CRI is the preferable approach as it results in stable depth of sedation along with reduction of required drug doses. Moreover, the profound cardiovascular effects following bolus administration tend to stabilize over time during the CRI $[13,14]$. Morphine is a pure agonist opioid analgesic which was successfully used as CRI in combination with repeated xylazine boluses and medetomidine in horses undergoing various standing surgical procedures [1517]. Based on the previously reported merits of $\alpha_{2}$-agonists/ 
opioid combinations along with importance of xylazine as the most economically and extensively used sedative agent for horses in Egypt, the present study aimed to evaluate the effects of a CRI of xylazine alone or in combination with morphine on sedation, analgesia and ataxia in horses.

\section{Materials and Methods}

\section{Animals}

Five adult mixed breed horses (four males and one female) aged $15.60 \pm 5.77$ years and weighing $286.33 \pm 16.01 \mathrm{~kg}$ were enrolled in this study. The horses were considered healthy and free from painful conditions based on physical examination, complete blood count and serum biochemistry analyses. This study was approved by the Ethical Committee of the Faculty of Veterinary Medicine, University of Sadat City. For the entire duration of the study, the horses were kept in their stall (provided with hay with free access to water) belonging to University of Sadat City.

At the end of the day prior to experiment, horses were weighed and the hair over right jugular vein was shaved for placement of venous catheter. Food but not water was withheld from horses for 12 hours before the experiment. On the day of the study, horses were kept in a stock in a quiet room and allowed at least two hours to acclimatize to their surroundings. Fly repellent was also applied to the skin of the horses to decrease external stimulation. The right jugular vein was catheterized under local anesthesia with subcutaneous lidocaine $2 \%(2 \mathrm{~mL})$ using a 16-gauge catheter (45mm, Harsoria health care, Haryana, India). Jugular catheter was connected to a three-way stopcock valve (Ghatwary medical Co, Alex, Egypt) that was secured in position for connection of the two infusion sets utilized for CRI of the studied drugs. Horses were subjected to two consecutive sedation protocols with a week wash out period in between. Subsequently, two treatment groups were evaluated.

Xylazine (XYL) Group: Horses received an intravenous bolus of xylazine at dose of $0.55 \mathrm{mg} / \mathrm{kg}$ followed by a CRI of $1.08 \mathrm{mg} / \mathrm{kg} /$ hour. After 5 minutes, about $10 \mathrm{~mL}$ saline bolus was administered over 2 minutes followed by CRI of saline. The CRI of xylazine and saline were conducted at a rate of $1 \mathrm{drop} /$ second through separate infusion sets attached to a three-way stopcock valve.

Xylazine/Morphine (XYL/MOR) Group: Horses received an intravenous bolus of xylazine at dose of $0.55 \mathrm{mg} / \mathrm{kg}$. Thereafter, a CRI of xylazine $(0.66 \mathrm{mg} / \mathrm{kg} /$ hour $)$ was started. About 5 minutes later an intravenous bolus of morphine was injected over 2 minutes at dose of $0.15 \mathrm{mg} / \mathrm{kg}$ diluted in $0.9 \%$ saline to a volume of $10 \mathrm{~mL}$. Immediately afterwards, CRI of $0.1 \mathrm{mg} / \mathrm{kg} /$ hour of morphine was started at a rate of $1 \mathrm{drop} /$ second.

\section{Assessments}

During the study, degree of sedation and analgesia was assessed at 10,15 minutes following xylazine administration and later every 15 minutes for 120 minutes during the CRI of the studied drugs and every 15 minutes after stopping the infusion for 60 minutes (135, 150, 165 and 180 minutes after xylazine administration).

\section{Evaluation of the Degree of Sedation}

Table 1: The multifactorial sedation scale (MFSS) used to score degree of sedation (head drop $\%$ and the horse response to auditory, visual and tactile stimuli).

\begin{tabular}{|c|c|c|}
\hline Variable & Score & Description \\
\hline \multirow[t]{4}{*}{ Head Drop \% } & 0 & No head lowering (head height was equal or higher than baseline) \\
\hline & 1 & Head slightly lowered $(<40 \%)$ \\
\hline & 2 & Head moderately lowered (40-60\%) \\
\hline & 3 & Head markedly lowered $>60 \%$ \\
\hline \multirow[t]{4}{*}{ Response to Auditory Stimulation } & 0 & Horse raised its head briskly and ears were erected or laid back \\
\hline & 1 & Horse calmly raised its head \\
\hline & 2 & Ear twitched or horse moved slightly \\
\hline & 3 & No observable response \\
\hline \multirow[t]{4}{*}{ Response to Visual Stimulation } & 0 & Undiminished response, animal move away vigorously \\
\hline & 1 & Muted response or subdued reaction and movements \\
\hline & 2 & Reaction significantly subdued (elevates head slightly) \\
\hline & 3 & No signs of visual arousal \\
\hline \multirow[t]{4}{*}{ Response to Tactile Stimulation } & 0 & $\begin{array}{l}\text { Rapid and marked response (head shake) to stimulation. It was also awarded when test } \\
\text { was extremely difficult to be performed }\end{array}$ \\
\hline & 1 & The response was easily elucidated but was slower \\
\hline & 2 & The response was sluggish and only elicited by prolonged stimulation \\
\hline & 3 & No response \\
\hline Total Score of Sedation Degree & $0-3$ & No sedation \\
\hline
\end{tabular}




\begin{tabular}{|c|c|c|}
\hline & $4-6$ & Mild sedation \\
\hline & $7-9$ & Moderate sedation \\
\hline & $10-12$ & Intense sedation \\
\hline
\end{tabular}

Degree of sedation was assessed based on determination of the head drop percentage. At each recording time, head drop percent was estimated by measuring the change in distance from the most ventral bony portion of the chin to the ground (in centimeters) with respect to baseline head height using a measuring tape attached to chin region $[8,17,18]$. For determination of baseline head height, the horses were observed for 15 minutes before drug administration and the most frequently observed head position was recorded as the baseline head height. A numerical rating scale ranged from 0-3 was used to score the degree of head drop at each observation time using a modification of scoring system previously listed by [17] (Table 1). Degree of sedation was also assessed by evaluating the response of studied horses to auditory, visual and tactile stimuli. Auditory stimulation was performed by clapping hands approximately 60 centimeters from the horse head according to [19]. The horse response to the used stimulus was subsequently scored using a scale modified from [3]. Visual stimulation was done by waving a piece of cloth towards the animal head with scoring of its reaction using scoring system previously described by $[20,21]$. Tactile stimulation was carried out by touching inside the pinna of the ear using pen with subsequent scoring for the resultant response using a scale modified from Clarke et al. $[9,11]$.
The behavioral response of the studied horses to different imposed stimuli (auditory, visual and tactile) was subjectively scored from 0-3 (Table 1). For overall scoring of the degree of sedation exhibited by each animal at each observation period, the score of head drop as well as the scores of horse response to auditory, visual and tactile stimuli were summated to develop a final multifactorial sedation scale (MFSS) as presented in Table 1.

\section{Evaluation of the Degree of Analgesia}

Analgesic effect was assessed depending on the animal response to two nociceptive stimuli, needle prick and electrical stimulation. In needle prick method, a 22-gauge, 1.25-inch needle was thrusted to its whole length just once at the right and left flank regions, alternatively after aseptic preparation of the proposed area. The animal response to needle prick was graded from 1-5 based on a numerical rating scale (NRS) (Table 2) adapted from [3]. Electrical stimulation method was based on determination of electrical nociceptive threshold. To evaluate the nociceptive threshold, an electrical stimulus $(100 \mathrm{~Hz}$ and $1 \mathrm{~ms})$ was applied to the horse skin at the shoulder region via two adhesive electrodes connected to electric muscle stimulator device (Healthtronic muscle stimulator JH4207, China).

Table 2: Numerical rating scale (NRS) used to assess analgesic effect in needle prick method.

\begin{tabular}{|c|c|}
\hline NRS & Response to Pinprick \\
\hline 1 & Dramatic response (the horse was alert and moved, kicked, or bit) \\
\hline 2 & Moderate response (the horse raised its head briskly and ears were erect or laid back) \\
\hline 3 & Slight or barely perceptible response (the ear twitched or horse moved slightly) \\
\hline 4 & No observable response \\
\hline 5 & \\
\hline
\end{tabular}

The shaved skin over shoulder region was washed with water and soap and degreased twice with alcohol and dried before attaching the electrodes of the muscle stimulator. The electrodes were placed $7 \mathrm{~cm}$ apart and secured in position using a sheet of adhesive and transparent medical tape. At each observation period, the intensity of electrical stimulus was gradually increased and intensity of the stimulus at which the animal turned its head back towards the stimulus (avoidance response) was recorded and considered as the end point (electrical nociceptive threshold). The displayed output of the electric muscle stimulator unit does not reflect the exact voltage of the electrical stimulus. Therefore, the electrodes of the unit were subsequently connected to an oscilloscope (FLUKE-PM 3394A, Holland) to determine the voltage corresponding to each recorded electrical stimulus throughout the study period.

\section{Evaluation of Ataxia}

Table 3: Numerical rating scale used to assess degree of ataxia.

\begin{tabular}{|c|c|}
\hline NRS & Degree of Ataxia \\
\hline 0 & No ataxia \\
\hline 1 & Mild ataxia. the horse was stable but slightly swaying \\
\hline 2 & Intense ataxia. The horse was leaning against the stock and swaying with its hind limbs crossed and its fore- \\
\hline 3 & limbs buckling at the carpal joints \\
\hline
\end{tabular}


Degree of ataxia was evaluated at different time points by using the numerical rating scale [22]. At each time point the animal was given a score ranged from 0-3 as listed in Table 3. To enhance reliability of the used scales for measuring the degree of sedation and analgesia, performance of the adopted methods and the assessment of the horses, response were conducted by the same observer who was unaware of treatment given. Degree of ataxia was also evaluated by same observer.

\section{Statistical Analysis}

Statistical analysis was performed with SPSS 16.0 software (SPSS, USA). The parametric variables involving head drop percent and electrical nociceptive threshold were analyzed using a oneway analysis of variance (ANOVA) with Dunnett's post-test for comparisons of means within each group in relation to baseline. Comparisons between groups at each time were performed with one-way ANOVA followed by Tukey's test. The Friedman test was used for the non-parametric variables including response scores to auditory, visual and tactile stimuli as well as sedation, ataxia and needle prick scores. The results of parametric variables were expressed as mean \pm standard deviation whereas non-parametric results were expressed as the median (range). The level of significance was set at $\mathrm{p}<0.05$.

\section{Results}

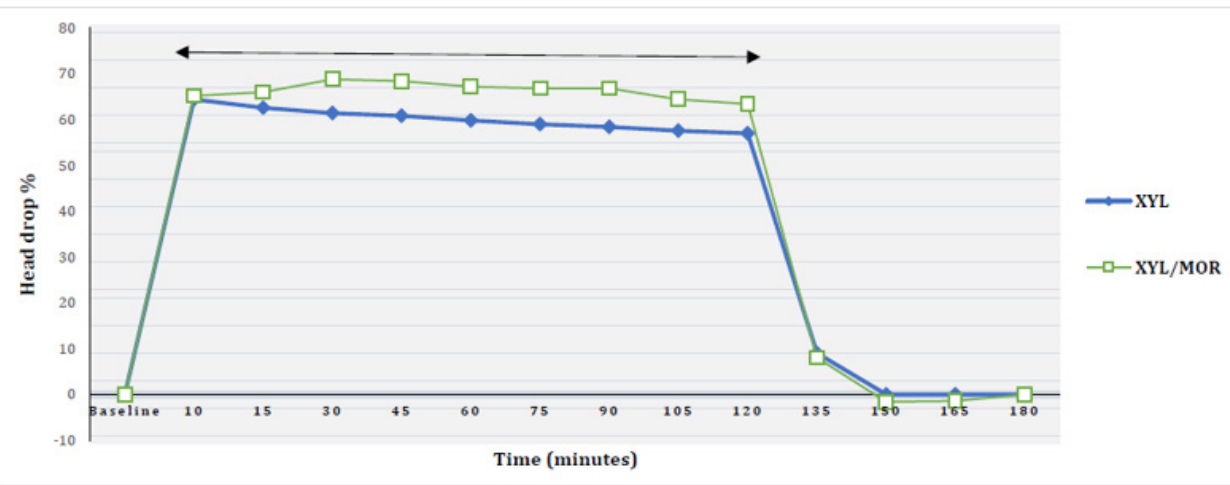

Figure 1: The mean values of head drop percent in horses after receiving a constant rate infusion of xylazine either alone (XYL) or combined with a constant rate infusion of morphine (XYL/MOR). $\leftrightarrow$ Significant difference between baseline and both treatments.

In both studied groups, the classic symptoms of sedation (head dropping, eyelid ptosis, ataxia and ear tip and lip separation) were evident just after xylazine administration. Considering the observed alterations in head height, head was significantly dropped below baseline from 10 minutes till 120 minutes in XYL and XYL/MOR groups. The maximum head drop percent was demonstrated at 10 minutes in XYL group $(64.40 \pm 4.04)$ and at 30 minutes in XYL/MOR group (68.80 \pm 6.06$)$. Head drop percent did not show significant differences in between treatments however it tended to be lower in XYL/MOR group than xylazine group till 120 minutes (Figure 1). Assessment of the horses, reaction to auditory, visual and tactile stimulation revealed that, both protocols induced a significant reduction in the response of horses to the used stimuli from 10 minutes up to 120 minutes relative to baseline. Significantly lower reaction to auditory stimulation was also evident in XYL/MOR group than XYL group at 30 minutes however significant difference was not detected among groups considering their response to visual and tactile stimuli (Table 4). Despite of this, slightly lower response to tactile stimulation was documented in XYL/MOR compared to XYL group. Regarding the degree of sedation, from 10 up to 120 minutes, significantly deeper sedation was demonstrated in all treatment groups than baseline. For the 120 minutes infusion period, moderate sedation was the most observable sedation whereas in XYL group, it was evident in all horses for 120 minutes, in XYL/MOR group, it was recorded in all horses at 10,15,90, 105 and 120 minutes and in four horses at other times (as intense sedation was evident in one horse).

Table 4: Median (range) of response scores to auditory, visual and tactile stimuli and sedation scores in horses after receiving a constant rate infusion of xylazine either alone $(X Y L)$ or combined with a constant rate infusion of morphine (XYL/MOR).

\begin{tabular}{|c|c|c|c|c|c|c|c|c|c|c|c|c|c|c|c|}
\hline \multicolumn{16}{|c|}{ Time (minutes) } \\
\hline Variables & Treatment & Baseline & 10 & 15 & 30 & 45 & 60 & 75 & 90 & 105 & 120 & 135 & 150 & 165 & 180 \\
\hline $\begin{array}{l}\text { Response } \\
\text { to auditory } \\
\text { stimulation }\end{array}$ & XYL/MOR & $1(0-2)$ & $2(2-3)$ & $\begin{array}{l}2(2-3) \\
2(2-3)\end{array}$ & $\begin{array}{l}2(2-2) \\
3(3-3)^{\delta}\end{array}$ & $\begin{array}{l}2(2-3) \\
3(2-3)\end{array}$ & $3(2-3)$ & $\begin{array}{l}2(2-3) \\
3(2-3)\end{array}$ & $\begin{array}{l}2(2-2) \\
3(2-3)\end{array}$ & $\begin{array}{l}2(1-3) \\
3(2-3)\end{array}$ & $\begin{array}{l}2(1-3) \\
3(2-3)\end{array}$ & $\begin{array}{l}1(0-2) \\
1(0-2)\end{array}$ & $\begin{array}{l}1(0-2) \\
1(0-2)\end{array}$ & $\begin{array}{l}1(0-2) \\
1(0-2)\end{array}$ & $\begin{array}{l}1(0-2) \\
1(0-2)\end{array}$ \\
\hline
\end{tabular}




\begin{tabular}{|c|c|c|c|c|c|c|c|c|c|c|c|c|c|c|c|}
\hline $\begin{array}{l}\text { Response } \\
\text { to visual } \\
\text { stimulation }\end{array}$ & $\begin{array}{c}\text { XYL } \\
\text { XYL/MOR }\end{array}$ & $0(0-1)$ & $2(1-2)$ & $\begin{array}{l}2(1-2) \\
2(1-2)\end{array}$ & $2(1-2)$ & $1(1-2)$ & $2(1-2)$ & $2(1-2)$ & $\begin{array}{l}2(2-2) \\
2(1-2)\end{array}$ & $\begin{array}{l}2(1-2) \\
1(1-2)\end{array}$ & $\begin{array}{l}2(1-2) \\
1(1-2)\end{array}$ & $\begin{array}{l}0(0-1) \\
0(0-1)\end{array}$ & $\begin{array}{l}0(0-1) \\
0(0-1)\end{array}$ & $\begin{array}{l}0(0-1) \\
0(0-1)\end{array}$ & $\begin{array}{l}0(0-1) \\
0(0-1)\end{array}$ \\
\hline $\begin{array}{l}\text { Response } \\
\text { to tactile } \\
\text { stimulation }\end{array}$ & $\begin{array}{c}\text { XYL } \\
\text { XYL/MOR }\end{array}$ & $\begin{array}{l}0(0-1) \\
0(0-1)\end{array}$ & $\begin{array}{l}1(1-2) \\
2(1-3)\end{array}$ & $\begin{array}{l}1(1-2) \\
2(1-3)\end{array}$ & $\begin{array}{l}1(1-2) \\
2(1-3)\end{array}$ & $\begin{array}{l}1(1-2) \\
2(1-3)\end{array}$ & $\begin{array}{l}1(1-2) \\
2(1-3)\end{array}$ & $\begin{array}{l}1(1-2) \\
2(1-3)\end{array}$ & $\begin{array}{l}1(1-2) \\
2(1-2)\end{array}$ & $\begin{array}{l}1(1-2) \\
2(1-3)\end{array}$ & $\begin{array}{l}1(1-2) \\
2(1-2)\end{array}$ & $\begin{array}{l}0(0-1) \\
0(0-1)\end{array}$ & $\begin{array}{l}0(0-1) \\
0(0-1)\end{array}$ & $\begin{array}{l}0(0-1) \\
0(0-1)\end{array}$ & $\begin{array}{l}0(0-1) \\
0(0-1)\end{array}$ \\
\hline $\begin{array}{l}\text { Sedation } \\
\text { score }\end{array}$ & XYL & $1(0-3)$ & $\begin{array}{c}8(7-9) \\
9(8-9)^{\delta}\end{array}$ & $\begin{array}{c}8(7-9) \\
9(8-9)^{\delta}\end{array}$ & $\begin{array}{c}8(7-8) \\
9(9-11)^{\delta}\end{array}$ & $\begin{array}{c}8(7-8) \\
9(9-11)^{\delta}\end{array}$ & $\begin{array}{c}8(7-8) \\
9(8-10)^{\delta}\end{array}$ & $\begin{array}{c}8(7-8) \\
9(8-10)^{\delta}\end{array}$ & $\begin{array}{l}8(7-8) \\
9(8-9)^{\delta}\end{array}$ & $\begin{array}{c}8(7-8) \\
9(8-9)^{\delta}\end{array}$ & $\begin{array}{l}8(7-8) \\
9(8-9)^{\delta}\end{array}$ & $\begin{array}{l}1(1-4) \\
1(1-4)\end{array}$ & $\begin{array}{l}1(0-3) \\
1(0-3)\end{array}$ & $\begin{array}{l}1(0-3) \\
1(0-3)\end{array}$ & $\begin{array}{l}1(0-3) \\
1(0-3)\end{array}$ \\
\hline
\end{tabular}

Note: Shaded timepoints indicate significant difference $(p<0.05)$ from baseline. ${ }^{\delta}$ Significant difference between $X Y L / M O R$ and $X Y L$.

Within each group, the highest sedation scores were obtained at 10 and 15 minutes in XYL and at 30 and 45 minutes in XYL/ MOR. In between treatments, sedation scores were significantly higher in XYL/MOR group compared to XYL group till 120 minutes (Table 4). With respect to analgesic effect of the studied treatments, significant increase in response scores to needle prick as well as electrical nociceptive threshold was demonstrated in all groups from 10 till 120 minutes relative to baseline. Despite lack of significant difference between groups, using both nociceptive models a slightly higher analgesic effect was evident in XYL/MOR group than XYL group at some time points. Comparing both models, all groups produced similar baseline scores to needle prick at 135 minutes while slight analgesic effect (not significant) was evident using electrical stimulation at 135 minutes in XYL and at 135,150 and 165 minutes in XYL/MOR group. Data in Table 5 also illustrated the presence of significantly higher ataxia scores in both groups than baseline at 10 and 15 minutes. At these time points, mild ataxia was exhibited by four horses in XYL group with moderate ataxia in the remaining one while in XYL/MOR group, mild ataxia was evident in two horses with moderate ataxia in three horses. Throughout the study, degree of ataxia did not vary significantly in between groups.

Table 5: The Median (range) of response scores to needle prick, Mean \pm SD values of electrical nociceptive threshold and Median (range) of ataxia scores in horses after receiving a CRI of xylazine either alone (XYL) or combined with a CRI of morphine (XYL/MOR).

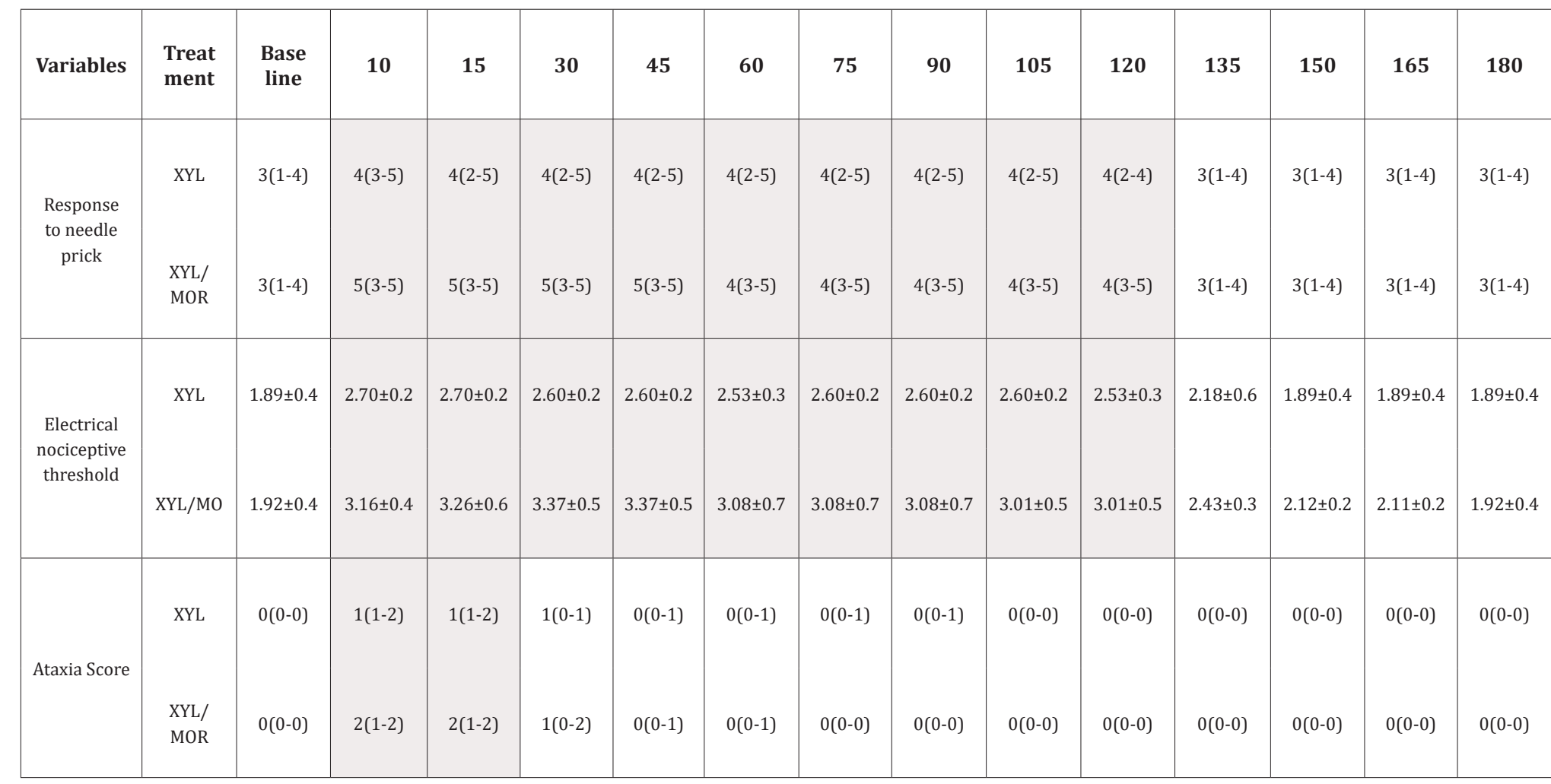

Note: Shaded timepoints indicate significant difference $(p<0.05)$ from baseline. 


\section{Discussion}

Due to increasing trend towards the use of standing sedation in horses for performance of various interventions and due to the necessity for reliable standing protocols with minimal side effects this study was established to evaluate and compare a morphine CRI/low xylazine CRI with high xylazine CRI. Following administration of $\alpha_{2}$-agonists, the classical signs of sedation (head lowering and dropping of eyelids, dropping of ears and lower lip) were recorded by many authors [18,23-26]. Consistently in the current study, similar symptoms were showed by all horses shortly after xylazine administration. Observable sedation achieved by $\alpha 2$-agonists can be mediated by their binding to $\alpha_{2}$ a-receptors located in the locus coeruleus of the pons which subsequently blocks norepinephrine release and produces sedation $[27,28]$. Regarding the degree of sedation, head drop percent was reported to be satisfactory to evaluate the sedative effects of $\alpha_{2}$-agonists when given alone or in combination with opioids $[17,18]$. However in another reports, using of head height alone was not sufficiently sensitive to determine the beneficial effect of adding butorphanol to romifidine and xylazine in horses [29,30]. Whereas this variable can be an indicator of degree rather than quality of sedation [31].

Therefore, in the study reported here, for elaborating a more reliable scale in elucidating the difference between sedative effect of the studied combinations, we designed MFSS that included assessment of alteration of head height as well as the horse's reaction to tactile, auditory and visual stimuli as common variables for sedation quality [32].The use of the three different stimuli was also intended to decrease the subjectivity of the scale. Considering XYL protocol (intravenous bolus of $0.55 \mathrm{mg} / \mathrm{kg}$ followed by a CRI of $1.08 \mathrm{mg} / \mathrm{kg} /$ hour), the used bolus was based on the study of de Souza et al. [22] while the CRI was selected due to the ability of this rate to maintain moderate sedation in the studied horses (unpublished work by our laboratory). Evaluation of head height as the first variable for sedation degree revealed the ability of the used XYL protocol to induce and maintain significant head drop during the infusion period. Consistently, significant reduction in head height was reported using approximately similar xylazine protocol (intravenous bolus of $0.55 \mathrm{mg} / \mathrm{kg}$ followed by a CRI of $1.1 \mathrm{mg} / \mathrm{kg} /$ hour) [22].

Similar data was described using $1 \mathrm{mg} / \mathrm{kg}$ xylazine bolus followed by a CRI of $0.69 \mathrm{mg} / \mathrm{kg} /$ hour [20]. In XYL/MOR group, a CRI of morphine was combined with $0.66 \mathrm{mg} / \mathrm{kg} / \mathrm{hour}$ of xylazine (unpublished data). This combination was not only able to achieve marked head drop compared to baseline, but also slightly greater drop compared to XYL group. On contrary, significantly lower head drop percent was reported when detomidine was combined with morphine compared to detomidine alone at some time points [18]. The discrepancy between both studies could be due to a slightly higher morphine bolus used in our study which might increase its ability to enhance the sedative effect of xylazine or allow a synergistic sedative effect between drugs to be evident. In XYL group, the studied horses exhibited reduced response to different imposed stimuli. In agreement with these findings was reported during xylazine CRI [20]. Pronounced depression of horses, reaction to auditory stimulation with slightly greater depression of their response to tactile stimulation was determined in XYL/ MOR group than XYL group. Collectively, the used MFSS seemed sensitive to determine the difference in the sedative effect between $\mathrm{XYL}$ and XYL/MOR protocols whereas this scale demarcated presence of significantly deeper sedation in XYL/MOR group than XYL group despite absence of significant difference in head drop percent. Superior sedation observed in XYL/MOR group could be resulted from potentiation of xylazine associated sedation by coadministration of morphine or synergism between drugs. In the same way, morphine $(0.1 \mathrm{mg} / \mathrm{kg})$ was found to be successful in improving the sedative effects of intravenous detomidine $(10 \mathrm{mg} / \mathrm{kg}$ ) in horses [33]. To evaluate the analgesic effect of the studied treatments, two nociceptive tests (needle prick and electrical stimulation) were conducted. Needle prick method was selected as being able to differentiate the analgesic properties of xylazine and xylazine/tramadol combination [10]. Regarding electrical stimulation, it is a commonly used method to investigate antinociception produced by analgesics in horses [34]. This method also seemed satisfactory to compare the analgesic effect of each of xylazine and detomidine given alone or combined with tramadol and to compare the analgesic effect of xylazine CRI with/out a CRI of lidocaine $[10,22,35]$.

To evaluate the electrical nociceptive threshold, electrical stimulus was applied to shoulder region of horses instead of limbs as ataxia observed with $\alpha_{2}$-agonists may increase the number of false negative responses as the animal may not respond even if it experiences pain [26,36]. Furthermore, opioids may cause excitement and locomotor stimulation in conscious and pain-free horses which may hinder the observer's interpretation of the response to the stimulus applied to the limb where the response is lifting the limb $[37,38]$. Also, to increase reliability of the used electrical test, the distance between the electrodes of the electric muscle stimulator device was standardized throughout the study to avoid possible changes in resistance that could affect the current on which stimulus intensity depends [36]. Both nociceptive test models, was easy to use and allowed determination of the analgesic effect of the studied treatments. The used models elucidated the analgesic effect of xylazine in horses. Consistently, xylazine associated analgesia was detected in different studies $[3,10,23,39]$. The attained analgesia can be explained by a direct spinal action of $\alpha_{2}$-agonists on $\alpha_{2}$-adrenoceptors which initiates pre-and postsynaptic inhibitory mechanisms [40,41]. Pre-synaptically, the release of neurotransmitter norepinephrine is inhibited while post-synaptically hyperpolarization of the cell membrane prevents further transmission of stimuli [40]. Supraspinal sites of action are also proposed for analgesia produced by $\alpha_{2}$-agonists [42]. Co-administration of morphine with xylazine resulted in slightly improved analgesia compared to using xylazine alone but they were not statistically different. The lack of significant difference could be attributed to the lower infusion rate used in XYL/MOR group 
compared to XYL group. Another cause could be the short-term nociception produced by needle prick and electrical stimulation methods whereas, nociception resulted from short-term electrical stimulation responds less to opioid analgesics than prolonged pain which involves an inflammatory component in the processing of nociceptive stimuli [43]. Therefore, despite the lack of significance observed under this experimental condition, we could speculate that, XYL/MOR combination could be the superior combination under clinical conditions as the animal would suffer from a more prolonged pain. Ataxia was a frequently reported complication following xylazine administration to horses [20,30]. Considering this and due to mild ataxia reported mainly following xylazine bolus $(0.55 \mathrm{mg} / \mathrm{kg})$ [22], we selected to use similar bolus dose of xylazine in order to limit its deleterious ataxic effect as the greatest ataxia usually occur in the time points close to administration of boluses. The obtained results revealed that this selection was adequate as moderate ataxia was the greatest level of ataxia that was encountered and was only detected in one horse in xylazine group. Furthermore, according to our results we speculated that tested xylazine protocol could have lesser ataxic effect compared to that previously used by [20]. Addition of morphine to xylazine caused slightly higher ataxia compared to xylazine alone however both groups were not significantly different. In agreement with these findings, morphine slightly increased the medetomidine induced ataxia in horses [17]. Despite of this, moderate ataxia was also the greatest degree of ataxia detected in this group which considered to be acceptable during standing procedures in horses [17].

\section{Conclusion}

Xylazine/morphine treatment was efficient to produce superior sedative effect and slightly better analgesic properties compared to xylazine treatment despite using lower xylazine infusion rate in combination with morphine. Based on this along with dosedependent side effects of $\alpha_{2}$-agonists, this protocol might be superior and safer alternative to xylazine protocol during standing interventions in horses.

\section{Acknowledgments}

The authors wish to thank the staff within their department for taking care of the examined animals during the study period.

\section{Conflict of Interest}

No conflict of interest

\section{References}

1. Le Blanc PH (1991) Chemical restraint for surgery in the standing horse. Vet Clin North Am Equine Pract 7(3): 521-533.

2. Bidwell LA, Bramlage LR, Rood WA (2007) Equine perioperative fatalities associated with general anesthesia at a private practice- a retrospective case series. Vet Anaesth Analg 34(1): 23-30.

3. Wagner AE, Mama KR, Contino EK, Ferris DJ, Kawcak CE (2011) Evaluation of sedation and analgesia in standing horses after administration of xylazine, butorphanol, and subanesthetic doses of ketamine. J Am Vet Med Assoc 238(12): 1629-1633.
4. Clarke KW, Trim CM, Hall LW (2014) Anesthesia of the horse in Veterinary Anesthesia. Saunders, Oxford: United Kingdom 266-268.

5. Alitalo I (1986) Clinical experiences with Domosedan (R) in horses and cattle a review. Acta Veterinaria Scandinavica.

6. England GK, Clarke KW, Goossens LA (1992) Comparison of the sedative effects of three $\alpha 2$-adrenoceptor agonists (ranitidine, detomidine and xylazine) in the horse. J Vet Pharmacol Ther 15(2): 194-201.

7. Corletto F, Raisis AA, Brearley JC (2005) Comparison of morphine and butorphanol as pre-anaesthetic agents in combination with romifidine for field castration in ponies. Vet Anaesth Analg 32(1): 16-22.

8. Rezende M, Grimsrud KN, Stanley SD, Steffey E, Mama K (2015) Pharmacokinetics and pharmacodynamics of intravenous dexmedetomidine in the horse. J Vet Pharmacol Ther 38(1): 15-23.

9. Clarke K, England GCW, Goossens L (1991) Sedative and cardiovascular effects of romifidine alone and in combination with butorphanol, in the horse. J Vet Anaesth18(1): 25-29.

10. Seo JP, Son WG, Gang S, Lee L (2011) Sedative and analgesic effects of intravenous xylazine and tramadol on horses. J Vet Sci 12(3): 281-286.

11. Gozalo-Marcilla M, Luna SP, Crosignani N, Puoli Filho JN, Possebon FS, et al. (2017) Sedative and antinociceptive effects of different combinations of detomidine and methadone in standing horses. Veterinary anesthesia and analgesia 44(5): 1116-1127

12. Vigani A, Garcia-Pereira FL (2014) Anesthesia and analgesia for standing equine surgery. Vet Clin North Am Equine Pract 30(1): 1-17.

13. Bettschart-Wolfensberger R, Bettschart R, Vainio O, Marlin D, Clarke K (1999) Cardiopulmonary effects of a two hour medetomidine infusion and its antagonism by atipamezole in horses and ponies. J Vet Anesth 26(1): 8-12.

14. Bettschart-Wolfensberger R, Clarke KW, Vainio O, Aliabadi F, Demuth D (1999) Pharmacokinetics of medetomidine in ponies and elaboration of a medetomidine infusion regime which provides a constant level of sedation. Res Vet Sci 67(1): 41-46.

15. Yaksh T, Noueihed RJ (1985) The physiology and pharmacology of spinal opiates. Annu Rev Pharmacol Toxicol 25(1): 433-462.

16. Ruiz J, Zuluaga D, Ruiz I, Duque D, Ochoa M, et al. (2015) Evaluation of sedation and analgesia protocols with xylazine and two rates of morphine by continuous infusion in stationary horses undergoing laparoscopic castration. Archivos De Medicina Veterinaria 47(3): 333340.

17. Solano AM, Valverde A, Desrochers A, Nykamp S, Boure LP (2009) Behavioural and cardiorespiratory effects of a constant rate infusion of medetomidine and morphine for sedation during standing laparoscopy in horses. Equine Vet J 41(2): 153-159.

18. Guilhen RC, Cassu RN, Diniz MS, Giuffrida R, Duarte RR A (2015) Comparison of detomidine in combination with saline, morphine or methadone in horses submitted to experimental oral stimuli. Semina: Ciências Agrárias 36(2): 4225-4235.

19. Love EJ, Taylor PM, Murrell J, Whay HR, Waterman-Pearson AE (2011) Assessment of the sedative effects of buprenorphine administered with $20 \mu \mathrm{g} / \mathrm{kg}$ detomidine in horses. Vet Rec 168:(15) 409.

20. Ringer SK, Portier K, Torgerson PR, Castagno R, Bettschart-Wolfensberger $\mathrm{R}$ (2013) The effects of a loading dose followed by constant rate infusion of xylazine compared with romifidine on sedation, ataxia and response to stimuli in horses. Vet Anaesth Analg 40(2): 157-165.

21. Peștean C, Oana L, Ober C, Miclauş V, Oros D (2010) Assessment of the sedative effects of xylazine, detomidine and romifidine in horses. Annals of RSCB 5(1): 282-284. 
22. Fernandes de Souza JF, Monteiro ER, Campagnol D, Ramos RC, Frasson AMF (2012) Evaluation of nociception, sedation, and cardiorespiratory effects of a constant rate infusion of xylazine alone or in combination with lidocaine in horses. J Equine Vet Sci 32(6): 339-345.

23. Moens Y, Lanz F, Doherr MG, Schatzmann U (2003) A comparison of the antinociceptive effects of xylazine, detomidine and romifidine on experimental pain in horses. Vet Anaesth Analg 30(3): 183-190.

24. Figueiredo JP, Muir WW, Smith J, Wolfrom G (2005) Sedative and analgesic effects of romifidine in horses. International Journal of Applied Research in Veterinary Medicine 3: 249-258.

25. Hewes CA, Keoughan GC, Gutierrez-Nibeyro S (2007) Standing enucleation in the horse: a report of 5 cases. Can Vet J 48(5): 512-514.

26. Valverde A (2010) Alpha-2 agonists as pain therapy in horses. Vet Clin North Am Equine Pract 26(3): 515-532.

27. Scheinin M, Schwinn DA (1992) The locus coeruleus: site of hypnotic actions of $\alpha 2$-adrenoceptor agonists? Anesthesiology 76(6): 873-875

28. Sinclair MD (2003) A review of the physiological effects of $\alpha 2$-agonists related to the clinical use of medetomidine in small animal practice. Can Vet J44(11): 885.

29. Ringer SK, Portier KG, Fourel I, Bettschart-Wolfensberger R (2012) Development of a romifidine constant rate infusion with or without butorphanol for standing sedation of horses. Vet Anaesth Analg 39(1): 12-20.

30. Ringer SK, Portier KG, Fourel I, Bettschart-Wolfensberger R (2012) Development of a xylazine constant rate infusion with or without butorphanol for standing sedation of horses. Vet Anaesth Analg 39(1): $1-11$.

31. Hamm D, Turchi P, Jöchle W (1995) Sedative and analgesic effects of detomidine and romifidine in horses. Vet Rec 136(13): 324-327.

32. Schauvliege S, Cuypers C, Michielsen A, Gasthuys F, Gozalo-Marcilla M (2019) How to score sedation and adjust the administration rate of sedatives in horses: a literature review and introduction of the Ghent Sedation Algorithm. Vet Anaesth Analg 46(1): 4-13.

33. Clarke KW, Paton BS (1988) Combined use of detomidine with opiates in the horse. Equine Vet J 20(5): 331-334
34. Spadavecchia C, Arendt-Nielsen L, Spadavecchia L, Mosing M, Auer U, et al. (2017) Effects of butorphanol on the withdrawal reflex using threshold, suprathreshold and repeated subthreshold electrical stimuli in conscious horses. Vet Anaesth Analg 34(1): 48-58.

35. Kim T, Jang M, Lee H, Lee I (2012) Sedative and Analgesic Effects of Intravenous Detomidine and Tramadol on Horses. Korean Journal of Clinical Veterinary Medicine 29(3): 226-232.

36. Lopes C, Luna S, Rosa A, Quarterone C, Crosignani N, et al. (2016) Antinociceptive effects of methadone combined with detomidine or acepromazine in horses. Equine Vet J 48(5): 613-618.

37. Carregaro AB, Luna SP, Mataqueiro MI, de Queiroz-Neto A (2007) Effects of buprenorphine on nociception and spontaneous locomotor activity in horses. Am J Vet Res 68(3): 246-250.

38. Love EJ, Taylor PM, Murrell J, Whay HR (2012) Effects of acepromazine, butorphanol and buprenorphine on thermal and mechanical nociceptive thresholds in horses. Equine Vet J 44(2): 221-225.

39. Rohrbach H, Korpivaara T, Schatzmann U, Spadavecchia C (2009) Comparison of the effects of the alpha- 2 agonists detomidine, romifidine and xylazine on nociceptive withdrawal reflex and temporal summation in horses. Vet Anaesth Analg 36(4): 384-395.

40. Yaksh TL (1985) Pharmacology of spinal adrenergic systems which modulate spinal nociceptive processing. Pharmacol Biochem Behav 22(5): 845-858.

41. Hamalinen MM, Pertovaara A (1995) The antinociceptive action of an $\alpha 2$-adrenoceptor agonist in the spinal dorsal horn is due to a direct spinal action and not to activation of descending inhibition. Brain research bulletin 37(6): 581-587.

42. Guo TZ, Jiang JY, Buttermann AE, Maze M (1996) Dexmedetomidine injection into the locus ceruleus produces antinociception. Anesthesiology 84(4): 873-881.

43. Le Bars D, Gozariu M, Cadden SW (2001) Animal models of nociception. Pharmacol Rev 53(4): 597-652. 